\title{
The PMA action via metabolism of uremic erythrocytes
}

\author{
D. Polak-Jonkisz ${ }^{\mathrm{a}, *}$, D. Zwolinska ${ }^{\mathrm{a}}$ and L. Purzyc $\mathrm{c}^{\mathrm{b}}$ \\ ${ }^{a}$ Department of Pediatric Nephrology, Wroclaw Medical University, ul. Borowska, Wroclaw, Poland \\ ${ }^{\mathrm{b}}$ Department of Medical Biochemistry, Wroclaw Medical University, Wroclaw, Poland
}

Keywords: RBCs, Caspase-3, PKC, chronic kidney disease

Dear Editor,

In the recent article Carelli-Alinovi $\mathrm{C}$ et al. [1] are raising an important issue of the link, established between PKC and caspase 3 activities and its association with NO metabolism. The authors made a normal, mature red blood cell (RBC) the object of their observations and analysis. Phorbol ester (PMA - phorbol12-myristate-13-acetate) activates protein kinases $\mathrm{C}$ (PKC) and these kinases phosphorylate a number of cytoskeletal and cytoplasmic proteins, e.g. band-3 or endothelial NO synthase (eNOS), localised in the cytoplasmic membrane of RBC [3]. PMA stimulates the production of reactive oxygen species (ROS) by plasma membrane NADPH (nicotinamide adenine dinucleotide phosphate) oxidase activation. These processes play a significant role in maintaining the mechanical and morphological properties of the RBC membrane. In our ex vivo studies of uremic erythrocytes, we also found $\mathrm{PKC}$ activation, which could have been released either during erythrocyte maturation or under the influence of biochemical factors, e.g., uremic toxins or oxidative or mechanical stress [unpublished data]. In line with the authors, we found a certain drop in the activity of eNOS, that may have resulted from calmodulin effects, which we used to observe in the course of chronic kidney disease and not merely from phosphorylation of the $\mathrm{Ca}^{2+}$-binding domain. In their manuscript, the authors seek to explain the role of PKC in the activation of caspases [1]. These proteases are involved in the degradation process of membrane proteins, among others, band-3, modulating its biological functions. Caspase-3 may be induced either directly or indirectly via a series of events between caspase-3 and PKC. Thus, perhaps, it is PMA which, whilst inducing oxidative stress, activates caspase-3, although the mechanism is not very clear. The authors are trying to explain the $\mathrm{PMA} \longrightarrow \mathrm{PKC} \longrightarrow$ caspases $\longrightarrow$ cellular membrane sequence, but they do not take into account the role of free, cytoplasmic calcium $\left(\mathrm{Ca}^{2+}{ }_{\mathrm{i}}\right)$, the concentration of which in physiological RBCs is many times lower than outside of erythrocytes. It may then be treated as an intracellular "signalling" molecule, the

\footnotetext{
${ }^{*}$ Corresponding author: Dorota Polak-Jonkisz, MD, PhD, Department of Pediatric Nephrology, Wrocław Medical University, ul. Borowska 213, 50-556 Wroclaw, Poland. E-mail: dorota.polak-jonkisz@umed.wroc.pl.
} 


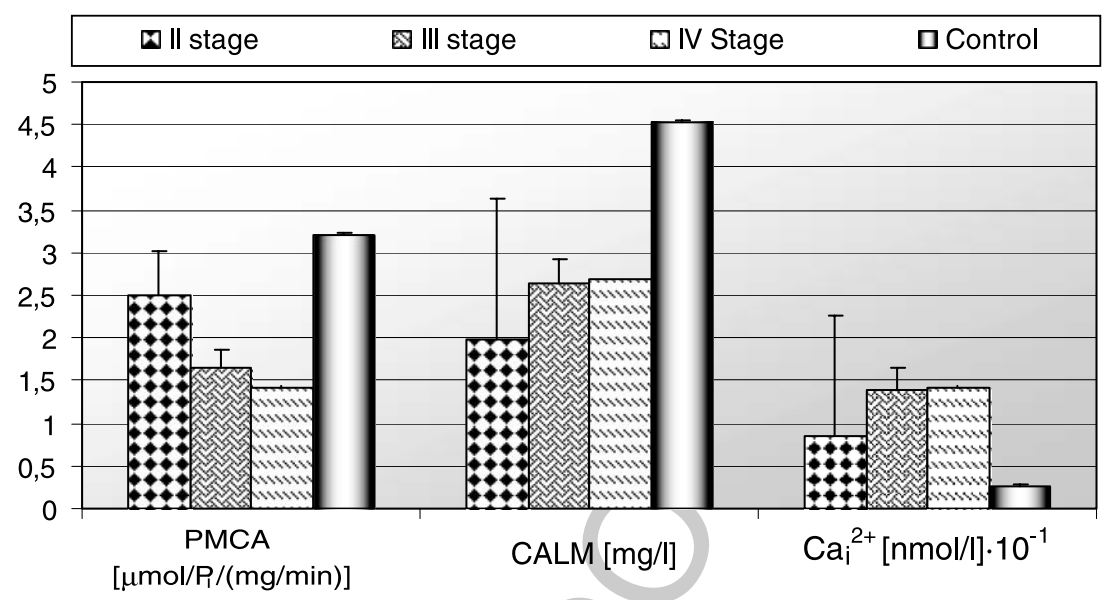

Fig. 1. The mean values of PMCA, CALM and $\mathrm{Ca}^{2+}$ in red blood cells in children with CKD stages II-IV and control group.

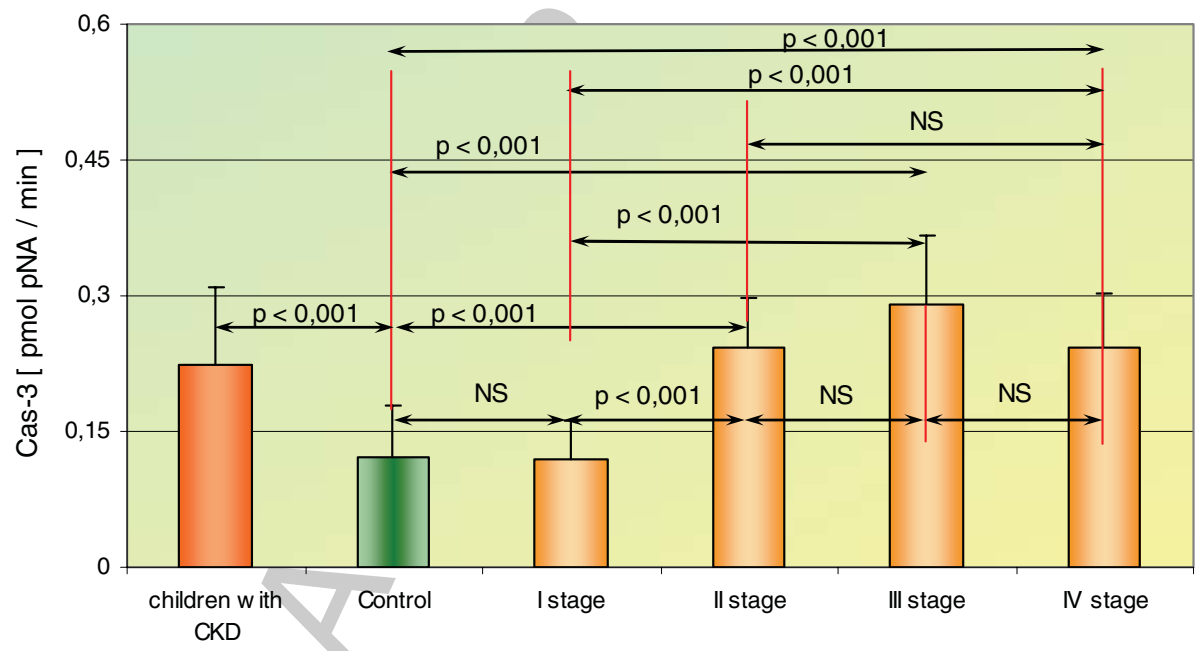

Fig. 2. The mean values of Casp-3 activity ${ }^{+}$in red blood cells in children with CKD stages I-IV and control group.

concentration changes of which affect, either directly or indirectly, the physiological function of cells, e.g.: cell injury, proliferation or eryptosis [2,4] (Fig. 1). The maintenance of this concentration gradient requires a precise control of the proteins, participating in the influx or efflux of calcium ions through the cytoplasmic membrane. The main protein, participating in the efflux of calcium ions through RBC membrane, is $\mathrm{Ca}^{2+}$ - dependent ATPase (PMCA). This pump directly depends on the activity of two proteases: caspases and the calpain-calpastatin complex. Their mutual $\mathrm{Ca}^{2+}{ }_{\mathrm{i}}$ concentration dependent correlations and interactions with proteins of the cytoplasmic membrane, are currently under broad discussion.

The modification of band-3 structure by caspase consists in its N-terminal domain being cut off, what contributes to dissociation of enzymatic glycolytic complexes and enhances glycolytic flux and, thus, also the ATP synthesis. The sufficiently high ATP concentration is decisive for the energetic level of RBC, being 
simultaneously, a substrate for PMCA and PKC, controlling the effects of their activity. Following CarelliAlinovi C. et al., glucose deficit and long-term exposure of cells to PMA, induces PKC translocations to the internal membrane side, thus stimulating its activity [1]. It results in increased phosphorylation of band-3 protein. In our studies of uremic erythrocytes, we observed a reducing tendency of the ATP concentration with a simultaneous increase of caspase 3 activity in the terminal stages of chronic kidney disease (Fig. 2). In our opinion, the cell protects itself against ATP deficit by enhancing glycolytic flux in result of the direct attack on band-3 [4]. The role of PKC translocation and of band-3 phosphorylation and the combined activity of the caspase-3 -calpain/calpastatin, as well as their correlations demand a separate research project.

Our observations in uremic erythrocytes and the above-mentioned study of Carelli-Alinovi et al., complement each other, thus extending our knowledge on erythrocyte metabolism disorders in disease, both in vitro and ex vivo. It should explain the process of ageing and premature death of RBC in a number of medical conditions.

\section{References}

[1] C. Carelli-Alinovi, D. Pirolli, B. Giardina and F. Misiti, Protein kinase C mediates caspase 3 activation: A role for erythrocyte morphology changes, Clin Hemorheol Microcirc (2014), doi: 10.3233/CH-141845

[2] F. Lang and S.M. Qadri, Mechanisms and significance of eryptosis, the suicidal death of erythrocytes, Blood Purif 33 (2012), $125-130$.

[3] C. Partovian, Z. Zhuang, K. Moodie, M. Lin, N. Ouchi, W.C. Sessa, K. Walsh and M. Simons, PKC alpha activates eNOS and increases arterial blood flow in vivo, Circ Res 97 (2005), 482-487.

[4] D. Polak-Jonkisz, L. Purzyc, M. Szczepańska and I. Makulska, Erythrocyte caspase-3 levels in children with chronic kidney disease, Clin Biochem 46 (2013), 219-224. 